This item was submitted to Loughborough's Research Repository by the author.

Items in Figshare are protected by copyright, with all rights reserved, unless otherwise indicated.

\title{
Partnership and privacy - tension or settlement? The case of adult mental health services
}

PLEASE CITE THE PUBLISHED VERSION

PUBLISHER

(c) Cambridge University Press

VERSION

VoR (Version of Record)

LICENCE

CC BY-NC-ND 4.0

\section{REPOSITORY RECORD}

6, Perri, Christine Bellamy, Charles Raab, and Adam P. Warren. 2019. "Partnership and Privacy - Tension or Settlement? the Case of Adult Mental Health Services". figshare. https://hdl.handle.net/2134/4494. 
This item was submitted to Loughborough's Institutional Repository (https://dspace.lboro.ac.uk/) by the author and is made available under the following Creative Commons Licence conditions.

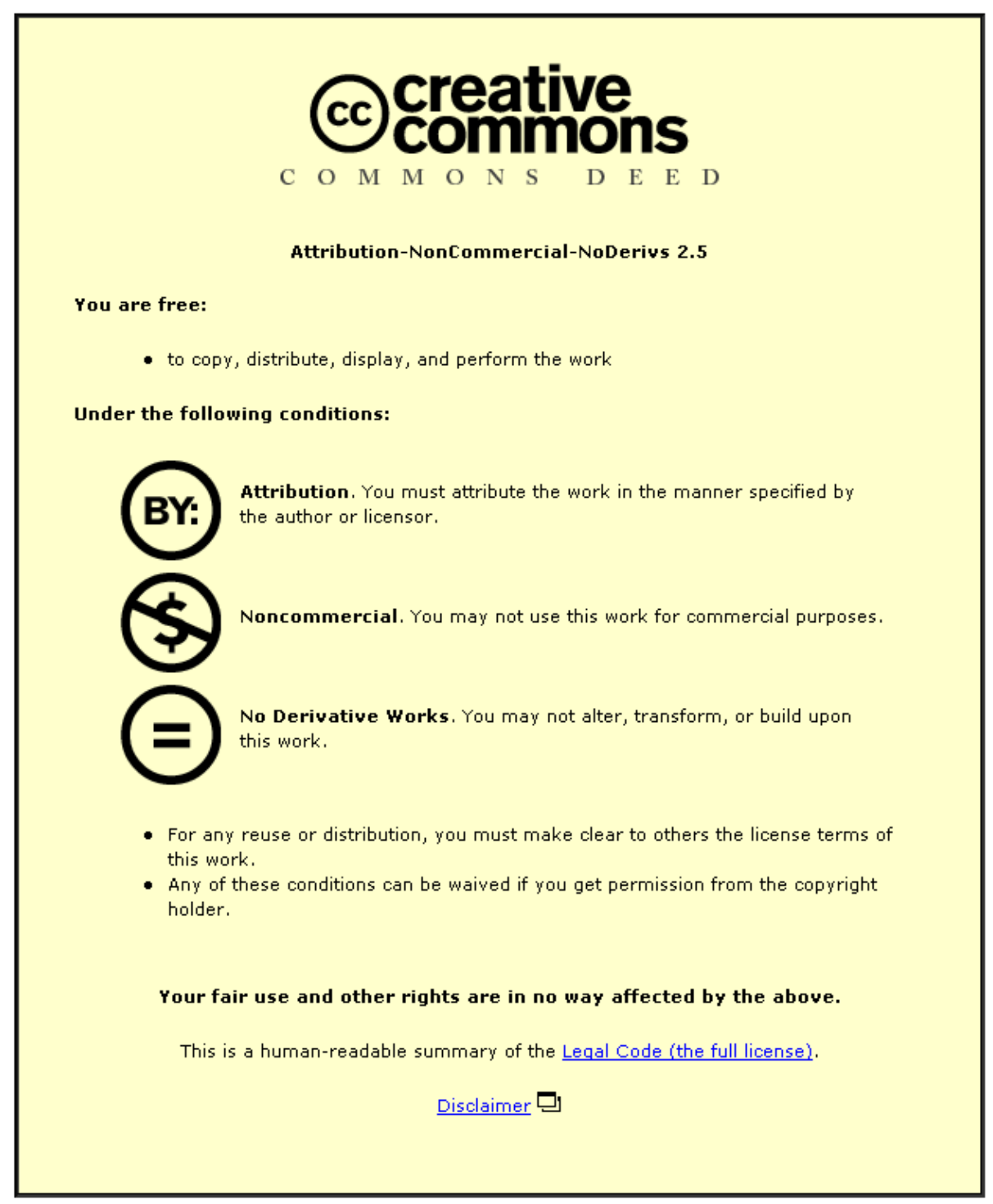

For the full text of this licence, please go to: http://creativecommons.org/licenses/by-nc-nd/2.5/ 


\title{
Partnership and Privacy - Tension or Settlement? The Case of Adult Mental Health Services
}

\author{
Perri 6*,1, Christine Bellamy ${ }^{2}$, Charles Raab ${ }^{3}$ and Adam Warren ${ }^{4}$ \\ ${ }^{1}$ Professor of Social Policy, Nottingham Trent University \\ E-mail Perri.6@ntu.ac.uk \\ ${ }^{2}$ Professor of Public Administration and Associate Dean (Research and Graduate Studies), Nottingham Trent University \\ ${ }^{3}$ Professor of Government, School of Social and Political Studies, University of Edinburgh \\ ${ }^{4}$ Research Fellow, Department of Information Science, Loughborough University
}

Mental health is a good example of a field where imperatives for partnership or collaborative working can be in tension with those for client confidentiality. Both imperatives have been reinforced by additional regulation in recent years, in response to major inquiries. Professionals face the dilemma that either sharing clients' or patients' information or not sharing it could lead to outcomes for which they might be blamed; any rule adopted risks one or other type of error. This article examines two cases from a larger interview-based study of how local organisations are trying practically to reconcile these competing pressures.

\section{Introduction}

In social policy and administration, important tensions can arise between the imperatives of partnership working between agencies and those of client confidentiality. Although these are not new problems, they have been given added salience by the introduction of electronic systems that make the technical aspects of information sharing in partnerships and networks easier to solve. In turn, technological advances make it more, rather than less, necessary to focus on organisational barriers to inter-agency data sharing in a more explicit fashion than when it was possible to hide behind feasibility problems (6 et al., 2005, Bellamy et al., 2005).

However, professionals and public managers do not have to make a stark choice between one value and entirely ignoring or overriding the other. A variety of settlements are possible, but the problem for frontline workers and their managers is that there is simply no obvious metric offering robust and unchallengeable solutions to each and every case. The government has intervened increasingly strongly over the last few years, and national policy directives, codes of practice and guidelines have proliferated, but there is no avoiding the need to exercise professional judgment in street level work. We know, however, very little about how frontline workers exercise such judgments, and what factors shape them. In this article, we therefore draw upon data from a major ESRC-funded programme of qualitative research, designed to address that knowledge gap. ${ }^{1}$ The study 
covers a wider range of multi-agency partnerships in mental health, care of older people, public protection (i.e., those agencies dealing with dangers posed by sexual and violent offenders) and crime reduction.

Failures on the part of agencies working in such fields to share information from client records have been blamed for a long line of scandals going back decades. Inquiries into child deaths from neglect or abuse have typically pointed the finger at weaknesses in information sharing between social services, primary health care, law enforcement, education and sometimes other agencies: in this respect, at least, little changed between the reports into the deaths of Maria Colwell (Field-Fisher, 1974) and of Victoria Climbié (Laming, 2003). The Bichard Inquiry (2004) into how lan Huntley - a man previously known to the Humberside police as someone suspected of sexual assaults upon women came to be employed at Soham Village College, where he murdered two young girls, focused in large part on the rules adopted by the Humberside force for the weeding of 'soft' information about suspects who were never convicted of an offence, and the lack of national capability among police forces to share information about suspects.

Similar phenomena can be found in mental health. The Ritchie (1994) inquiry into the much publicised case of Christopher Clunis, a paranoid schizophrenic who had lost touch with his support services and who killed a stranger at a London tube station made the same, familiar points about failures to share information made by many other mental health homicide reports over many decades (Butler and Drakeford, 2005). Mental health is a field in which issues of personal information are particularly fraught. Because mental illness has long been stigmatised, information about an individual's mental ill-health is usually restricted to those people who have the strongest 'need to know' about individuals' conditions. For much of the postwar period, patients' records from secondary psychiatric care services were subject to more restrictive practices of confidentiality than many other fields of health care. Because so many people vulnerable to acute episodes of mental illness were effectively sequestrated from the rest of society until the 1980s when the programme of closure of large psychiatric hospitals gathered pace, issues of information sharing were relatively simple: many were matters of joint referrals to secondary care and discharge to primary care agencies.

The development of Care in the Community in the late 1980s and the early 1990s introduced imperatives for sharing more types of information between more agencies. High profile failures of coordination of care and the loss of contact with people such as Clunis made the issue of information sharing critical to the politics of mental health. Conservative Governments repositioned Care in the Community from a policy focused on securing greatest benefits for people with mental health problems to one of managing the risks the most dangerous cases posed for the public. The preparation of risk management plans for discharge, and the maintenance of multi-agency control over those deemed to present a risk, especially to others, became increasingly central to the governance of mental health. Under the Labour government, the development of statutory Multi-Agency Public Protection Arrangements (MAPPAs) for managing violent and sexual offenders in the community have also involved psychiatric services in integrated partnership, working with offenders with personality disorders or mental health problems connected with their offending. This development, too, required additional sharing and confidentiality management with law enforcement and other social services. More generally, forensic, prison and probation-based mental health programmes have expanded their roles in recent years. 
A key aim of reform throughout the 1990s was to integrate services under a system of protocols known as the Care Programme Approach. This Approach was initially introduced at the beginning of the 1990s, but was subsequently developed and refined. These protocols specified the kind of information to be shared on a need-to-know basis and the agencies that might receive or provide it. They carefully emphasised the overriding authority of the common law of confidentiality and new data protection legislation $(\mathrm{DH}$, 1999a). They also specified the general powers of approved mental health social workers, medical staff and police officers: the three professions that until now have been involved in making what is often seen as the most difficult decision of all in mental health, namely, whether and when to authorise the compulsory detention of a person suffering an acute episode. Local agencies were urged to adopt detailed information sharing protocols ( $\mathrm{DH}$, 1999a, esp. para $45 \mathrm{ff})$.

In its first term, the Labour government took a fresh look at mental health $(6$ and Peck, 2004) and issued a National Service Framework setting out standards of care in England (DH, 1999b). In every part of England, Local Implementation Teams (LITs) were to be set up; health and social care agencies had to put in place joint arrangements for collaborative working toward integrated and seamless care. A mental health information strategy was subsequently published, seeking to put in place electronic arrangements for appropriate information sharing $(\mathrm{DH}, 2001)$. However, with the introduction of the NHSwide programme for new information technologies and electronic patients records known first as the National Programme for Information Technology (NPfIT) and later as Connecting for Health - the mental health programme was swept into the overall programme, but given a lower priority than more politically salient areas, such as the Choose and Book initiative for elective surgery. Being a low priority in the NHS is not an uncommon experience for mental health services, but, as a result, a certain siege mentality has grown up in some parts of the service; this has coloured the character of informal organisation in the field - a matter to which we shall return below.

If the termination of the separate mental health information strategy is intended to promote integration, then it could be welcomed. It is, of course, important to offer people with mental health problems good all-round health services and to enable those responsible for other aspects of health care to know as much as they need to know about any mental health problem that might impact on their work. There is undoubtedly a case for integrating mental health record systems with those of the rest of the NHS, subject to appropriate rules of confidentiality. However, the low priority attached to those aspects of systems specific to mental health has had a retarding impact on the development of integrated services in England, by contrast with what seemed to be possible in 2001 and 2002 when the government's mental health information strategy was published. At the time of writing, the electronic patient record programme is not fully implemented across the whole country, and, in particular, the mental health modules are not yet in place, although some pilots have been completed. This state of affairs holds broadly true, too, of the electronic social care record programme, which is of at least as great importance for mental health care as the NHS primary and secondary psychiatric care record systems. Certainly, in the years 2003-05, when the fieldwork for the research presented here was done, many mental health care services were still using some paper record systems, together with a variety of rather basic electronic ones. In this respect, the field has fallen behind even the police or criminal justice organisations, which have been severely castigated by the Bichard Inquiry in this regard. 
Health records were first declared to be 'sensitive' in law, as opposed to merely informally so, in the 1995 European Data Protection Directive, which was implemented in England by the Data Protection Act 1998 (s1.(2)). After serious criticisms in the 1990s of NHS confidentiality practices by the Data Protection Commissioner (now the Information Commissioner), a series of initiatives were undertaken by the Department of Health and more recently by what is now the Connecting for Health programme. These initiatives put in place systems and rules of confidentiality management for the NHS generally (Bellamy et al., 2005). In 2003, following consultation and public attitude research, the Department issued a Code of Confidentiality ( $\mathrm{DH}, 2003)$. This Code offered patients for the first time a clear opportunity to require certain categories of personal information not to be shared without further specific consent, save in emergencies when reasons for overriding refusal of consent would be logged. This facility was described in the consultation paper as a virtual 'sealed envelope'. Its importance for mental health records generally, and especially for primary care records concerned with mental health, was immediately clear. At least as important was the commitment to undertake a huge exercise across the NHS to tabulate the routine 'needs to know' of every type of professional post, so far as patient information is concerned, and to embody them in a set of restricted permissions to access relevant parts of electronic care records. This scheme is known as 'role-based access'. At the time of writing, this scheme has yet to be fully implemented in mental health care, not least because of the peculiar challenges of achieving it in Care Trusts where health and social care professionals work in integrated teams, but where there is as yet no national scheme for role-based access for social care professionals. In 2005, the Care Record Development Board in the Connecting for Health programme published a Care Record Guarantee (NHS, 2005) recommitting the service to the need-to-know principle for governing all sharing of patients' personal information. In addition, there are professional codes of conduct governing confidentiality in mental health services. Some are specific to particular professions and are issued by the professional institutes for psychiatry and other mental health professions, while others apply to doctors or nurses generally.

The management of patient information in mental health is thus now subject to additional imperatives for greater sharing - both to control risk to the public and to enhance the quality and integration of services. At the same time, there are now greater safeguards for confidentiality protection than was previously the case. This situation presents professionals and local services managers with demanding challenges to find workable settlements to the apparent tensions between these two imperatives.

Reasonably enough, many frontline staff and their managers are concerned about the possibility of being blamed either for sharing or not sharing, when decisions turn out later to lead to unwelcome outcomes. At the point where a decision on whether or not to share the information is being made, the professional's confidence in their judgment of risk must unavoidably be rather low, for neither the sending nor the receiving organisation possesses a full picture of the case. Indeed, the whole point of sharing information is to enable a more comprehensive view to be assembled, the better to assess risk. It follows that whether a decision to share is appropriate or inappropriate - and therefore beyond legal challenge - is one that it is actually impossible to make without the benefit of hindsight. If the decision to share turns out - because of subsequent events or in the light of aggregated information - to have been unnecessary, then the initial decision to share was a false positive judgment error that unnecessarily breached confidentiality. The converse error, an inappropriate decision not to share information, counts likewise as 
a false negative judgment error. If, having shared the information and conducted further investigations, the initial judgment error is not subsequently corrected, then inappropriate intervention may follow and the harm may be compounded. For example, a child may be wrongly taken into care, or a putatively mentally ill person may be unnecessarily compelled to be treated in a psychiatric unit ('sectioned'). As well as the potential for injustice and harm to vulnerable people, the problem facing street level professionals and bureaucrats is that any decision rule or norm applied under such conditions of limited and opaque information inevitably runs the risk either of false negative or of false positive judgment errors, and that sooner or later they will make the wrong judgment call, and thus open themselves to blame and to other adverse consequences to their reputations and careers.

Findings: how do frontline professionals deal with tensions between partnership working and privacy?

To understand how managers and professionals in mental health deal with apparent tensions between partnership working and privacy, we provide some illustrative interview evidence from two local case studies. They comprise two English Local Implementation Teams (LITs) set up under the National Service Framework to provide integrated care spanning health and social services for the mental health National Service Framework. As indicated above, these two cases are taken from a larger set of cases in several service delivery fields. Case study numbered 1 in our set of 12 cases, is a LIT in a mainly rural area that comprises 24 organisations, including a mental health trust, an NHS Care Trust, a local authority social services department and both voluntary and private sector agencies. The other case study, numbered 2, comprises 14 organisations. These include a mental health trust, a Primary Care Trust, individual GPs, a social services department, a local MIND organisation and other voluntary agencies, and several sheltered housing providers.

We found that both LITs relied heavily on paper records. Indeed, there were few electronic record systems used in case study 1 , although in the other, social services were piloting shared electronic records. There was much greater diversity among teams and organisations in 1 than in 2 about how the records were organised. Access rules for information that might be shared among teams and organisations were not well defined in either case. Indeed, there was a good deal of discretion about whether access to whole databases was granted, or access only to a record or part of a record. This discretion was supposed to be exercised in compliance with data protection law and national codes, but there was limited LIT level regulation of professionals' practices in this regard. Discretion was exercised by workers in NHS bodies so as to provide segmented access to parts of records on a 'need to know' basis, in broad compliance with NHS codes.

Space prevents deeper analysis of these cases and their theoretical implications. Evidence gathered for the project nevertheless throws interesting light on how frontline staff deal with tensions between data sharing and confidentiality. We were particularly interested in understanding how decisions to share or not to share client information are shaped by the social dynamics of organisational and inter-organisational working, especially the interplay between formal and informal institutional processes. To do this, we tested a number of hypotheses derived from neo-Durkheimian institutional 
theory associated with Mary Douglas and others (Douglas, 1982; Thompson et al., 1990; 6 et al., 2002). The theoretical framing of the project is set out in 6 et al. (2004, 2005).

\section{Institutional forms in mental health partnerships}

Perhaps our most striking finding is that, by contrast with the other case studies, the two mental health LITs were consistently more likely than were any of the other cases to show more traces of enclaving in the mix of institutional forms within the partnership. That is, many frontline professionals work in organisations or teams characterised by weak regulation by external bodies such as professional organisations or government departments, but which are strongly integrated within themselves. In mental health, multidisciplinary teams such as crisis resolution teams or assertive outreach teams, or some voluntary agencies, strongly exhibit the characteristics of enclaves. Such characteristics are also present at LIT level, especially in the large NHS bodies such as the mental health trusts, but rather less strongly and consistently. In contrast, these organisations also displayed hierarchical tendancies, where strong regulation and strong social integration combine to create high expectations of compliance with organisational and professional norms. The proliferation of government guidelines and the strong professionalisation of work in the mental health field probably accounts for the finding that the mental health LITs also showed fewer tendencies than, say, the police and other law enforcement agencies to individualist forms where weak external regulation combines with weak internal integration to create significant scope for enterprising individuals to broker arrangements for information sharing between teams, on the basis of personal relationships or trust. Mental health partnerships are rather more likely to exhibit isolate forms which combine high levels of regulation with weak social integration, thus making it difficult for frontline workers to do much more than cope as best they can with the exigencies of their work. In isolate cultures, workers are likely to distance themselves from efforts to improve organisational practices and even managers become fatalistic about confidentiality or data-sharing practices.

The significance of professional enclaves for information sharing in mental health partnerships

The relatively strong presence of enclaves in mental health LITs significantly effects the ways in which professionals deal with tensions between information sharing and privacy, because enclaved institutional forms shape information management practices in distinctive ways. Enclaved institutions typically impress upon their members the importance of marking boundaries between themselves and the rest of the world. In terms of information sharing practices, this means that they place high confidence in colleagues' willingness to maintain confidentiality within the enclave, and thus tend to encourage fairly free sharing of information among its members, even when those members belong to different professions. In contrast, members tend to distance themselves from other organisations in the partnership and become suspicious about their practices. For example, a manager of a psychiatry team in case study 1 commented that 
I guess I would see myself as belonging to [...] firstly the team and then probably under the locality and then the Trust and probably the kinda wider (X) bit of you know the LITs and the PCT (Primary Care Trust) etc.

Enclaves typically maintain their integration and mark their boundaries by stressing the internally egalitarian character of team working. These features are emphasised by one manager of a psychiatry-based team in case study 2, a team which is composed of people from several professions, each of very different status:

Erm, what we do is, we have a reflective practice model, so it means that first of all we do all the joint assessments, it's always two people either a nurse and a doctor, nurse and social worker, two nurses, whatever is feasible at the time. We do joint assessments, when we come to the end of the assessment we ask the client, usually to help you to get the client's view, then we say, 'Right, we're going to go and talk with the team'. The assessing team goes into the office and we all participate in decision making. We decide together, whoever is on duty ...

This manager also stressed the principled character of the collective decision making procedure adopted with respect to proposals or requests for sharing of client information beyond the team:

Whatever comes to information sharing, it is again the team that talks about it, we have to weigh the risks, the advantages, you know the whole works.

The overall tendency is to inhibit data sharing across the LIT, even when it is appropriate, and to make something of an obsession of confidentiality as a way of policing the boundaries of the enclaved team. A manager of the early-intervention-in-psychosis team in case study 1 describes well his sense of distance across the boundary between the team and the rest of the LIT:

I've been in [names LIT] for twenty five months now and I guess I suppose I don't have a huge amount of input with the Mental Health LIT. I don't actually have an awful lot to do with it, although I know I try and influence it to make sure intervention is a priority.

Boundaries in such enclaves are often drawn to include certain categories of clients, as well as staff, and this tendency also hinders the sharing of client information. A worker in an emergency psychiatric team, which often has to deal with mentally ill people in confused, distressed and, sometimes, dangerous states, complains of the unwillingness of the local Drug Action Team, a strongly enclaved team, to share data about their clients:

Well because of the nature of their work, [...] they've got very strict confidentiality rules between themselves and their clients. And unless the client has got a written statement with us here authorising us to get information from drug and alcohol teams they just wouldn't give us any information.

From the other side of the fence, a manager of an assertive outreach team in case study 1 talked of their dedication to their clients and the information held, including an objection to the use of generic consent forms to share information with other organisations. 
This sense of commitment to confidentiality on the basis of principle rather than the letter of regulatory prescription is particularly important in enclaved settings:

But, you see, signing a form that gives permission to share information with other agencies is like signing a blank cheque. Each case is different and if I was a patient I wouldn't sign any such form, I would be saying, 'Well what information do you want me to share and with whom?' So when, [...] it's required it should be discussed with the client, it's no good saying you've got a letter of consent in the file to share information and then share it with everyone else. That's not good, that's not clear in respect of confidentiality.

Enclaved institutional forms are particularly common in teams that are on the margins of LITs, and they tend both to result from and to reinforce distance and distrust. A manager of a charity-run assertive outreach team (case study 2) described their team's frustrations with the LIT, when asked about the barriers to data sharing presented by the LIT's formal administrative structure:

I sometimes, I'm trying to put this diplomatically erm, ... I do wonder at times how much the LIT are truly aware of the difficulties and the good work that really actually does go on in the Trust, but I don't know how in touch they really are, because they certainly never come and ask me anything. [emphasis added]

Conversely, LIT level staff in more hierarchical settings can be frustrated by the preciousness of enclaves about confidentiality, their excessive confidence about their internal information handling practices and their unwillingness to integrate with others in the LIT. A team manager told us:

I don't like the idea of teams having their own little confidentiality sort of thing, you know. We need to be mindful of confidentiality, and we need to be sharing information with other people within a fair framework of risk and we need to be mindful of people's confidentiality because it can break down. People can get free and easy.

As we should expect in a setting with a marked articulation of enclaved institutions within an overall mix that includes a significant hierarchical element, the forms of regulation of information sharing and confidentiality that are stressed as most important and acceptable are those which are based in professional codes, rather than those which flow from the organisation (either a Care Trust or else a conventional NHS trust or local authority social services department). The view of the manager of the Early Intervention in Psychosis team is quite typical in the assertion of collectively espoused principle as more authoritative than organisational prescription:

I'm pretty sure again I guess I take my confidentiality from my own, I think there are trust guidelines but I can't remember or even flicked through them, but I would have a very good idea of what was confidential and it would be about from my professional organisation and professional training which is about things can be broken only if you think people are at risk otherwise everything is confidential. [emphasis added] 

get this right?

Being unable directly to observe sharing and non-sharing behaviour, we asked interviewees how confident they were that acceptable practices were adopted in these case study partnerships, so far as confidentiality and appropriate information sharing were concerned. We hypothesised that enclaves would show more confidence in their own practices than in those of the rest of the organisation or LIT. We expected that this ambivalence will probably show up in moderate confidence in partnership working as a whole. Secondly, we expected that interviewees in strongly isolate forms would show less confidence in the information sharing and the confidentiality practices of their organisations than others. People working in isolate cultures therefore tend to distance themselves from the organisation in which they are employed, and to show qualified loyalty to its practices, including those designed to encourage partnership working. An element of hierarchy in the institutional mix - that is, strong regulation and strong integration, with reasonably clear rule-bound and role-based systems of authorisation was predicted to increase confidence that data are being shared appropriately, and that confidentiality is being properly respected.

Our evidence tends to confirm these hypotheses. In particular, the relatively high level of regulation - and particularly hierarchical institutional forms - in mental health accounts for the fact that we heard much less evidence of conflict within these LITs over confidentiality and sharing practices than we did in our other case studies, particularly those in the field of crime reduction and public protection. As expected, the enclaved character of some of the teams showed up as a powerful factor, in that interviewees generally had much less confidence in compliance with either sharing or confidentiality codes by teams or organisations other than their own. However, as we also expected, strong enclaves are more strongly associated with greater confidence in confidentiality being respected than that client data are being shared when it is appropriate for this to happen.

There was, however, marked variation between organisations within the LITs in levels of confidence that client information would be shared when it was appropriate to do so. In particular, there was a marked contrast between the greater confidence in compliance with information sharing norms exhibited by interviewees in the mental health trusts and the weaker confidence of those in the primary care trusts and social services. The weakest levels of confidence overall were shown by those in the voluntary bodies. This finding was consistently observed in our other case studied, too, but the contrast was rather less severe in relation to compliance with confidentiality norms. As might be expected, interviewees in organisations or teams exhibiting strong isolate characteristics tend to have lower confidence on both dimensions.

Interviewees were also asked whether they were more worried about being blamed for having shared or for not having shared information. In the present political climate, we expected that most would say that they were more worried about being blamed for not sharing. This expectation was generally borne out. However, a minority were concerned about being blamed for sharing and so violating confidentiality or else were equivocal. This finding probably reflects the deeply engrained confidentiality norms instilled into many frontline workers in this field, and is in line with the rather greater confidence in compliance with confidentiality norms than in information sharing across 
these partnerships. We were therefore particularly interested in finding out whether increased regulation - in the form of stronger pressure from central government expressed mainly through the issuing of policy documents and guidelines - was associated with more confidence in data-sharing practices, and whether it provided greater subjective security against the fear of blame. We found that, indeed, the stronger presence of hierarchy tends to be associated with increased confidence in both dimensions of data handling. However we also found that the proliferation of codes and rules simply adds to the problems of frontline workers, unless they have a strong, shared understanding of their purpose. In other words, the assertion of formal modes of regulation encourages confidence in information sharing only in so far as these modes are supported by equally strong informal modes, including those shaped by professional training, shared experience and a consistently applied policy. An interview with a manager of a day centre in Case 2 illustrates this point well:

Well I mean, the thing is, I'Il refer to psychological guidelines by the association, I would refer to social work guidelines. I'll refer to nursing guidelines .... We have got guidelines but we haven't got a policy.

\section{Conclusion: the implications for partnership working and citizenship}

Increased regulation, which has certainly produced pressure for hierarchy, consists in rules that cannot be turned mechanically into algorithms but still require individual professional judgment to interpret and apply those rules. What has happened is that the place of discretion and judgment has been shifted from the simple substantive task of deciding the merits of cases, to that of completing the formal decision rules where they are necessarily incomplete. Judgment remains central to professional practice, and in our mental health case studies, the need for it has, if anything, been reinforced, not reduced. Formal institutions are not sufficient to provide guidance in every case, but nor are they easy to supplement with craft knowledge.

However, exercising that judgment exposes the individual worker to the risk of public obloquy for judgment errors. In particular, the risk management agenda that took off under the previous Conservative Government, and has developed strongly since 1997 too, has generated growing pressure in favour of sharing, in order to bear down on false negative judgment errors.

The increase in top-down regulation has neither eliminated professional discretion and judgment nor reduced the scope for informal practices; indeed it may have increased their influence. The persistence of partially enclaved, and to a lesser degree of isolate institutional forms, appears to be a reaction, albeit an unintended one, to the subjective vulnerability of frontline workers to the assertion of a stronger but uncertain hierarchy. In mental health, the combination of additional regulation and the low priority accorded the sector, together with the growing problem of exposure to public blame whenever errors are made, nurtures a counter-reaction in the form of enclaving at team and organisation levels. The result may well be an unstable settlement, in which defensive practices drive responses to felt tensions between imperatives for sharing and confidentiality, especially where trust between teams and organisations is not strong enough to sustain the shared tacit knowledge that would enable formal rules to work consistently across partnerships. 
What, then, are the implications of our findings for the meaning of citizenship in the field of mental health? In the brief space left to us, we would make a number of points. The first is that these cases illustrate the complex issues at stake in the way we should understand relations between citizens and state in the light of the increased capabilities for processing personal information in late modern conditions. The discourse of the 'big brother' state tends to cast this problem in terms of a simple opposition between the growing surveillance capabilities of the state and the interests and rights of 'citizens'. The cases discussed here show, rather, that, in their everyday work, street level professionals are regularly forced to juggle two sets of rights and interests, each belonging to the same citizens, namely the citizens' right to privacy - a right that is now enacted in the UK in the Human Rights Act - and their interest in effective, joined-up service delivery, an interest that is now increasingly acknowledged by social policy. In the mental health field, this problem is compounded when a citizen presents risks to other citizens, especially when that risk is of a severity that cannot be assessed without inter-agency sharing of information to which the citizen may not be able or willing to consent.

In an ideal world, all citizens could expect these rights and interests to be recognised and weighed against each other in ways that are predictable and consistent, with the result that similar cases are treated similarly from time to time, place to place, organisation to organisation. The evidence from our project shows a particularly strong association across all our case studies between relatively high levels of regulation (as manifest in hierarchical institutional forms) and relatively high levels of satisfaction with confidentiality and information sharing practices amongst interviewees. We interpret this as indicating that many frontline professionals acknowledge imperatives to consistency of treatment and do not claim to maximise their personal discretion at its expense.

We have also seen, however, that there are good and inescapable reasons why consistency and predictability may be impossible to achieve in such fields, so far as resolving tensions between imperatives to information sharing and imperatives for confidentiality is concerned. By definition, decisions to share or not to share personal information about clients are taken in conditions of very imperfect information and are inherently prone to false positive or false negative judgment errors. A consistency of sorts might be achieved if the changing political climate induces a systematic bias in favour of tolerating more false positive errors in order to reduce the risks of false negative ones, by persuading frontline workers that they are more likely to be blamed for not sharing than for sharing. But the 'political climate' is notoriously fickle, and a high profile scandal of over-zealous sharing leading to a wrongful 'sectioning' or treatment could quickly swing the pendulum in the opposite direction again. Moreover, our evidence suggests that many frontline workers do not lightly reduce thresholds for overriding confidentiality norms. The achievement of even this limited kind of consistency is, in practice, mediated by the social dynamics of organisational life.

\section{Note}

1 'Joined-up public services: data sharing and privacy in multi-agency working', ESRC Research Award no RES/000/23/0158. The study involved 209 semi-structured interviews, conducted between 2003 and early 2005 with managers, professionals and information systems managers in local public service provision and purchasing agencies in eight local partnership arrangements across England involving 55 organisations and four in Scotland comprising 23 organisations, all in the policy fields of health and social 
care and crime and disorder; here only the findings are reported from the English case studies, focusing on just two. See 6 et al., 2005 for more detail.

\section{References}

6, P. and Peck, E. (2004), 'New Labour's modernisation in the public sector: a neo-Durkheimian approach and the case of mental health services', Public administration, 82, 1, 83-108.

6, P., Bellamy, C.A. and Raab, C.D. (2004), 'Data sharing and confidentiality: spurs, barriers and theories', 'Political Studies Association annual conference', Lincoln University, 5-8 April 04.

6, P., Leat, D., Selzter, K. and Stoker, G. (2002), Towards Holistic Governance: The New Reform Agenda, Basingstoke: Palgrave.

6, P., Warren, A.,Bellamy, C.,Raab, C., and Heeney, C. (2005), 'The governance of information sharing in networked public management', paper given at the Public Management Research Association 8th Public Management Research Conference, University of Southern California, Los Angeles, 29 Sept 05-1 Oct 05.

Bellamy, C., 6, P. and Raab, C. (2005), 'Joined-up government and privacy in the United Kingdom: managing tensions between data protection and social policy', Part II, Public Administration, 83, 2, 393-415.

Bichard Inquiry (2004), The Bichard Inquiry Report, London: Home Office.

Butler, I. and Drakeford, M. (2005) [2003]), Scandal, Social Policy and Social Welfare, 2nd edn, Bristol: Policy Press.

Department of Health (1999a), Effective Care Coordination in Mental Health Services: Modernising the Care Programme Approach - A Policy Booklet, London: Department of Health.

Department of Health (1999b), A National Service Framework for Mental Health: Modern Standards and Service Models, London: Department of Health.

Department of Health (2001), Mental Health Information Strategy, London: Department of Health.

Department of Health (2003), NHS Code of Confidentiality, London: Department of Health.

Douglas, M. (1982), 'Cultural bias', in M. Douglas, In the Active Voice, London: Routledge \& Kegan Paul.

Field-Fisher, T.G. (1974), Report of the Committee of Inquiry into the Care and Supervision Provided in Relation to Maria Colwell, London: Department of Health and Social Security.

Laming, Lord H. (2003), The Victoria Climbié Inquiry, London: Department of Health and Home Office.

NHS (2005), The Care Record Guarantee - Our Guarantee for NHS Care Records in England, London: NHS.

North Central London Strategic Health Authority (2005) 'The independent review into the care and treatment of Mr Anthony Hardy', North Central London Strategic Health Authority, London.

Ritchie, J. (1994), Ritchie Report: Report of the Inquiry into the Care and Treatment of Christopher Clunis, London: HMSO.

Thompson, M., Ellis, R., and Wildavsky, A. (1990), Cultural theory, Boulder, CO: Westview Press. 